\title{
Calciprotein particles are a sensitive marker predicting vascular calcification in patients with chronic kidney disease
}

Hidekazu Moriya ( $\nabla$ h_moriya@shonankamakura.or.jp )

Shonan Kamakura General Hospital

Yasuhiro Mochida

Shonan Kamakura General Hospital

Kunihiro Ishioka

Shonan Kamakura General Hospital

Machiko Oka

Shonan Kamakura General Hospital

Kyoko Maesato

Shonan Kamakura General Hospital

Sumi Hidaka

Shonan Kamakura General Hospital

Takayasu Ohtake

Shonan Kamakura General Hospital

Yutaka Miura

Jichi Medical University

Makoto Kuro-o

Jichi Medical University

Shuzo Kobayashi

Shonan Kamakura General Hospital

\section{Research Article}

Keywords: Calciprotein particle, Chronic kidney disease, Vascular calcification

Posted Date: December 15th, 2020

DOI: https://doi.org/10.21203/rs.3.rs-123757/v1

License: (1) (1) This work is licensed under a Creative Commons Attribution 4.0 International License.

Read Full License 
1 Calciprotein particles are a sensitive marker predicting vascular calcification in patients with

2 chronic kidney disease

3

4 Hidekazu Moriya $^{1)}$, Yasuhiro Mochida ${ }^{1)}$, Kunihiro Ishioka ${ }^{1)}$, Machiko Oka ${ }^{1)}$, Kyoko

5 Maesato $^{1)}$, Sumi Hidaka ${ }^{1)}$, Takayasu Ohtake ${ }^{1)}$, Yutaka Miura ${ }^{2)}$, Makoto Kuro-o ${ }^{2)}$, Shuzo

6 Kobayashi $^{1)}$

7

8 1) Department of Kidney Disease and Transplant Center, Shonan Kamakura General

9 Hospital, Kamakura, Kanagawa, Japan

10 2) Division of Anti-Ageing Medicine, Center for molecular Medicine, Jichi Medical

11 University, Shimotsuke, Tochigi, Japan

12

13 Corresponding author

14 Hidekazu Moriya

15 Department of Kidney Disease and Transplant Center, Shonan Kamakura General Hospital,

16 Kamakura, Kanagawa, Japan

17 Address: 1370-1, Okamoto, Kamakura, Kanagawa, Japan 247-8533

18 Phone: $+81-467-46-1717$

19 Fax: +81-467-45-9792

20 E-mail: h_moriya@shonankamakura.or.jp 
1 Abstract

2 Background: In chronic kidney disease (CKD) patients, vascular calcification occurs in the 3 early stage of CKD, before the titer of existing CKD mineral bone disorder (CKD-MBD)-

4 related markers exceed the threshold. Calciprotein particles (CPPs) are crystals in which

5 excessive phosphorus and calcium in the blood form colloidal particles and are associated

6 with vascular calcification. However, it is unknown whether CPPs are a more sensitive

7 marker for detecting calcification than others.

8 Methods: In a prospective cohort study of 58 patients with CKD we examined CKD-MBD

9 markers, including CPPs. Vascular arterial calcification score (ACS) of the lower extremities

10 was measured with the Agatston score. One year later, the markers and ACS were

11 reevaluated, and the relationship between the degree of progression of vascular calcification

12 and CKD-MBD markers was evaluated.

13 Results: The CPP titer was significantly correlated with that of serum phosphate and

14 corrected calcium. However, the CPP titer showed no correlation with eGFR or ACS in the

15 lower extremities. After one year, the basic titers of serum creatinine, eGFR, FGF-23, intact-

16 PHT, 1.25-dihydroxyvitamin D3 and CPP were not significantly correlated with ACS

17 changes in the lower extremities. There was a significant correlation between the rate of

18 change in ACS and that in CPP $(r=0.292, p=0.0258)$. CPP was an independent risk factor

19 for the progression of calcification in the lower extremities in a multivariate analysis ( $p=$

20 0.0144).

21 Conclusions: CPP is a more sensitive marker of arterial calcification than other CKD-MBD

22 markers in patients with CKD.

23 Key words: Calciprotein particle, Chronic kidney disease, Vascular calcification 


\section{Background}

2 Chronic kidney disease (CKD) is associated with mineral bone disorder (MBD) from an

3 early stage of CKD. CKD-MBD is not only related to bone changes and a biochemical

4 increase in phosphate, but also causes vascular calcification and arteriosclerosis, leading to

5 the onset of cardiovascular disease and poor prognosis. Generally, in stages 3-5 of CKD, the

6 optimal range of serum phosphorus concentration is set to be the normal reference value

7 (approximately $4.5 \mathrm{mg} / \mathrm{dl}$ or less) at each institution.

8 However, the phosphate level increases only when GFR falls below approximately 30

$9 \mathrm{~mL} / \mathrm{min} / 1.73 \mathrm{~m}^{2}$, at that which point vascular calcification, arteriosclerotic disease, and

10 lacunar infarction have already occurred [1,2]. It is too late to start intervention to control

11 CKD-MBD at that point.

12 Several hormones, including PTH, vitamin D, and FGF-23, are involved in the

13 metabolism of phosphate. FGF-23 acts as a phosphate diuretic hormone secreted by the bone

14 in response to the phosphate overload. The titer of FGF-23 is already increased before the

15 phosphate concentration exceeds the normal range. In addition, excess calcium and

16 phosphate in the blood are captured by mineral-binding proteins such as fetuin-A, which

17 forms calciprotein particles (CPPs). CPPs are microcrystals of calcium phosphate, and a

18 defense mechanism works to prevent these crystals from growing large. However, CPPs are

19 also considered to cause chronic inflammation and damage to the blood vessels, including in

20 various organs such as the heart and kidney [3].

21 FGF-23 and CPPs increase, and fetuin-A decreases, according to the progression of CKD

22 stages. In the CKD rat model, CPPs increased prior to aortic calcification [4], and it has been

23 reported that CPP is more strongly associated with ectopic calcification than fetuin-A [5].

24 However, it is unknown which markers of CKD-MBD are most sensitive to the formation of

25 arteriosclerosis in humans, and there are uncertainties regarding the correlation between the

26 titer of FGF-23 and that of CPPs in CKD.

27 FGF-23 is considered to increase from the point at which eGFR is $<75 \mathrm{~mL} / \mathrm{min} / 1.73 \mathrm{~m}^{2}$,

28 but if CPPs increase earlier, before FGF-23 changes, it could be possible to predict vascular

29 calcification lesions earlier and start therapeutic intervention by using CPPs as a marker of

30 vascular calcification.

31 In this study, we examined how CPPs associate with other markers of CKD-MBD and

32 vascular calcification in patients with $\mathrm{CKD}$.

34 Methods

35 Study design and population 
To determine the relationship between CKD-MBD markers and vascular calcification, we conducted a prospective cohort study in Shonan Kamakura General Hospital. The inclusion criteria were as follows: 1) consecutive recruitment between July 2014 and December 2016 from our clinic; 2) change in serum creatinine level within $0.3 \mathrm{mg} / \mathrm{dL}$ for the previous three months. The exclusion criteria were as follows: 1) refusal to provide informed consent; 2) contraindication of multidetector-row computed tomography (MDCT); 3) presence of hemodialysis, malignancy, congestive heart failure, and infections.

\section{Laboratory analyses}

10 We carefully obtained patients' history and laboratory data, including those concerning 11 serum creatinine, calcium, phosphate, C-reactive protein, intact-parathyroid hormone (i$12 \mathrm{PTH}), 1.25$-dihydroxyvitamin D3, urinary protein, fractional excretion of phosphate, and 13 FGF-23 levels. FGF-23 was measured using an intact FGF-23 ELISA kit (Kinos, Tokyo, 14 Japan) according to the manufacturer's protocols. All other samples were analyzed at the 15 local Department of Clinical Biochemistry in our hospital. Blood tests were performed again 16 after one year.

Examination by multidetector computed tomography

19 We calculated the Agatston score using MDCT (LightSpeed Ultrafast 16, General Electric 20 Medical System, Tokyo, Japan), in which the score is well correlated with that measured by electron-beam CT [1]. Volumetric data of the entire extremities were obtained in the helical mode with scanning parameters of $1.25 \mathrm{~mm}$ collimation width 16 detectors, a gantry rotation speed of $0.5 \mathrm{~s}$ per rotation, $120 \mathrm{kV}$, and $100 \mathrm{~mA}$. Images of $2.5 \mathrm{~mm}$ thickness with the center of the temporal window corresponding to $80 \%$ of the R-R interval were reconstructed with $2.5 \mathrm{~mm}$ spacing. Calcium score, volume, and mass were determined on a commercially available external workstation (Adventure Windows, version 4.4.1, General Electric Medical System, Tokyo, Japan) using CAC-scoring software (version 3.5, Smartscore, Tokyo, Japan), with MDCT. According to the Agatston method, we defined the regions of interest by vessel and slice with the threshold option for pixels greater than 130 Hounsfield units to measure the area and peak density of plaques. MDCT was conducted at the start of the study and after one year.

34 Quantification of CPPs in the plasma was performed as previously reported [6]. Briefly, a 35 fluorescent probe that binds to CaPi crystals (OsteoSense 680EX; PerkinElmer Inc., 
1 Waltham, MA) was added to heparin plasma samples. After incubation at $25^{\circ} \mathrm{C}$ for 60

2 minutes, the sample was applied to a gel-filtration spin column to remove unbound $\mathrm{CaPi}$

3 crystals. The fluorescent intensity of the flow-through was quantified using an infrared

4 fluorescence scanner (Odyssey CLx; LI- COR Biosciences, Lincoln, NE) and expressed as

5 an arbitrary unit (AU).

\section{Statistical analysis}

8 Continuous variables are presented as the mean \pm SD or median (interquartile range; IQR),

9 as appropriate. Spearman's correlation coefficients and multiple logistic regression analyses

10 were used as appropriate to test correlation between variables.

11 All analyses were conducted in SPSS Statistics 19. Results were considered significant if $12 P$ was $<0.05$.

\section{Results}

15 A total of $58 \mathrm{CKD}$ patients were included in the present study. Of these 58 patients, 24

$16(41.3 \%)$ had type 2 diabetes mellitus and $45(77.5 \%)$ had hypertension. The mean age of the

17 participants was $70.0 \pm 12.0$ years (45 men and 13 women). The basic characteristics of the

18 participants are shown in Table 1. Participants with CKD had a median creatinine level of

$191.42 \mathrm{mg} / \mathrm{dL}$ and a median eGFR of $39.7 \mathrm{~mL} / \mathrm{min} / 1.73 \mathrm{~m}^{2}$. The median FGF-23 level was 63.7

$20 \mathrm{pg} / \mathrm{mL}$ and the median CPP level was 16616 AU. The median ACS of the lower extremities was 655 (IQR: 0-706).

22 CPP level correlated well with serum phosphate and corrected calcium $(r=0.390 p$ $<0.0001, r=0.329 p<0.0001$, respectively). However, CPP level showed no significant relationship with eGFR (Figure 1A, 1B, 1C). CPP level also showed no significant relationship with age or FGF-23 level.

In univariate analyses, urinary protein and fractional excretion of phosphate correlated significantly with ACS in the lower extremities. However, these factors showed no correlation after adjusting for age and kidney function (Table 2). Smoking, diabetes mellitus, and systolic blood pressure were significantly correlated with calcification score in a multivariate analysis. No CKD-MBD markers, including CPPs, correlated with ACS in the lower extremities (Figure 2).

32 After one year, we reevaluated the calcification score and examined the relationship 33 between basic factors and the change in ACS. However, the change in calcification score 34 was not significantly correlated with any factors related to CKD-MBD (Table 3). In the next 35 step, when evaluating the relationship between the rate of change in calcification score and 
1 the rate of change in FGF-23, i-PTH, and 1.25-dihydroxyvitamin D3, there was no

2 significant correlation. However, the rate of change in CPP did show a significant

3 relationship with ACS in the univariate analysis $(r=0.292, p=0.0258)$, and in the multivariate

4 analysis when adjusting for age and kidney function $(r=0.298, p=0.0144)$ (Table 4).

\section{Discussion}

7 This study revealed that CPPs could be the only marker of CKD-MBD for assessing

8 changes in vascular calcification. Excessive calcium and phosphate in the blood are captured

9 by mineral-binding proteins such as fetuin-A to form CPPs, which are microcrystals of

10 calcium and phosphate. When calcium and phosphate exceed the solubility limit, the crystals

11 appear in the blood. Defense mechanisms work to prevent amorphous and primary CPPs

12 from growing into large and secondary CPPs in the extracellular space. However, when

13 CPPs become large, they cause vascular calcification in the heart, kidneys, brain, and lower

14 extremities. A small CPP containing only amorphous calcium phosphate is a physiological

15 CPP that induces the production of FGF-23 in osteoblasts, whereas a large CPP containing

16 calcium phosphate crystals causes cytotoxicity to vascular endothelial cells and renal tubular

17 cells, resulting in calcification in vascular smooth muscle cells [7]. It therefore becomes a

18 pathological CPP with the ability to induce hyperplasia and calcification in vascular smooth

19 muscle cells and innate immune responses in macrophages [8].

20 When a CPP is added to vascular smooth muscle cells, BMP-2 and osteopontin are

21 inducted and osteoblast-like changes occur in these cells. However, a high concentration of

22 phosphate alone does not induce similar changes in vascular smooth muscle cells. It is

23 therefore suggested that it is not phosphate but CPPs, formed by an overload of phosphate,

24 that cause vascular calcification [9]. In fact, in the presence of agents that inhibit the

25 formation of CPPs, an increase in phosphate concentration does not cause cell damage. In

26 the present study, the concentration of phosphate does not show a significant association

27 with the vascular calcification score of the lower extremities.

28 PTH, 1.25-dihydroxyvitamin D3, and FGF-23 are involved in mineral metabolism. FGF-

2923 is secreted from osteocytes/osteoblasts and acts as a hormone that increases urinary

30 phosphate excretion in the kidney in response to phosphate load from the diet and is a

31 counterregulatory hormone for 1.25-dihydroxyvitamin D3. The level of FGF-23 is increased

32 to adjust the phosphate level before the blood phosphate level exceeds the normal limit.

33 In this study, CPPs, rather than FGF-23, 1,25-dihydroxyvitamin D3, or intact PTH,

34 sharply predicted changes in vascular calcification score in the lower extremities, suggesting

35 that CPPs change earlier in response to mineral metabolism. 
The serum calcification propensity test $\left(\mathrm{T}_{50}\right)$ is an in vitro assay that quantifies the transformation time from primary to secondary CPP in the serum when challenged with exogenous calcium and phosphate. It has been used recently as an index of calcification risk. Low $\mathrm{T}_{50}$ values have been reported to be associated with all-cause mortality in patients with CKD and in kidney transplant patients and are also predictors of cardiovascular mortality [10-13]. However, it is reported that when adjusting for eGFR, there is no independent relationship between $\mathrm{T}_{50}$ and mortality in patients with CKD [14]. In addition, another report revealed that there is no direct relationship between vascular calcification and the values of $9 \mathrm{~T}_{50}[15]$.

10 Conversely, it has been reported that the accuracy of prognosis prediction might be 11 improved by evaluating the change in $\mathrm{T}_{50}$ via continuous measurement, rather than one point 12 measurement [16]. Regarding CPPs in this study, we were able to predict the progress of 13 vascular calcification by evaluating the change in CPP level, rather than using one point 14 measurement.

\section{Conclusion}

17 CPP level was not correlated with eGFR or vascular calcification score based on one 18 point measurement. However, change in the CPP level was significantly correlated with 19 change in vascular calcification. CPPs have a protective effect on vascular calcification by 20 trapping calcium and phosphate. Therefore, CPP is a more sensitive marker of arterial calcification than other CKD-MBD markers such as FGF-23.

23 Abbreviations

24 CKD: Chronic Kidney Disease; CKD-MBD: CKD Mineral Bone Disorder; CPP:

25 Calciprotein Particle; ACS: Arterial Calcification Score; eGFR: estimated Glomerular

26 Filtration Ratio; FGF-23: Fibroblast Growth Factor-23; PTH: Parathyroid Hormone; MDCT:

27 Multidetector-row Computed Tomography; BMP-2: Bone Morphogenetic Protein-2

\section{Declarations}

30 Ethics approval and consent to participate

31 The study protocol was approved by the Tokushukai Group Ethics Committee (TGE00434-

32 024). The study was conducted in accordance with the Declaration of Helsinki. Informed

33 consent was obtained from all subjects or, if subjects are under 18, from a parent and/or legal 34 guardian. 
1 Consent for publication

2 Not applicable.

3

4 Availability of data and materials

5 The datasets used and/or analyzed during the current study available from the corresponding

6 author on reasonable request.

8 Competing interests

9 The authors declare that they have no competing interests.

11 Funding

12 None.

14 Authors' contributions

15 HM coordinated the study, analyzed the data and wrote the manuscript. YM, KI, MO, KM,

$16 \mathrm{SH}, \mathrm{TO}, \mathrm{YM}$ and MK participated in the study design and coordination, analyzed the data.

17 SK is the guarantor of this work and, as such, had full access to all the data in the study and

18 takes responsibility for the integrity of the data and the accuracy of the data analysis. All

19 authors read and approved the final Manuscript.

21 Acknowledgments

22 Not applicable

24 Author details

25 1) Kidney Disease and Transplant Center, Shonan Kamakura General Hospital, 1370-1,

26 Okamoto, Kamakura 247-8533, Japan. 2) Division of Anti-Ageing Medicine, Center for

27 molecular Medicine, Jichi Medical University, Shimotsuke, Tochigi, Japan

29 References

30 1) Kobayashi S, Oka M, Maesato K, Ikee R, Mano T, Moriya H, et al. Coronary artery

31 calcification, ADMA, and insulin resistance in CKD patients. Clin J Am Soc Nephrol.

32 2008; 3: 1289-1295.

33 2) Kobayashi S, Ikeda T, Moriya H, Ohtake T, Kumagai H. Asymptomatic cerebral

34 lacunae in patients with chronic disease. Am J Kidney Dis. 2004; 44: 35-41. 
3) Kuro-o M. A phosphate-centric paradigm for pathophysiology and therapy of chronic kideny disease. Kidney Int Suppl. 2013; 3: 420-426.

4) Smith ER, Ford ML, Tomlinson LA, Rajkumar C, McMahon LP, Holt SG. Phosphorylated fetuin-A-containing calciprotein particles are associated with aortic stiffness and a procalcific milieu in patients with pre-dialysis CKD. Nephrol Dial Transplant. 2012; 27: 1957-1966.

5) Hamano T, Matsui I, Mikami S, Tomida K, Fujii N, Imai E, et al. Fetuin- mineral 21: 1998-2007.

6) Miura Y, Iwazu Y, Shiizaki K, Akimoto T, Kotani K, Kurabayashi M, et al. Identification and quantification of plasma calciprotein particles with distinct physical properties in patients with chronic kidney disease. Sci Rep. 2018; 8: 1256.

7) Akiyama K, Miura Y, Hayashi H, Sakata A, Matsumura Y, Kojima M, et al. Calciprotein particles regulate fibroblast growth factor-23 expression in osteoblasts. Kidney Int. 2020; 97: 702-712.

8) Kuro-o M. Klotho, phosphate and FGF-23 in ageing and distributed mineral metabolism. Nat Rev Nephrol. 2013; 9: 650-660.

9) Sage AP, Lu J, Tintut Y, Demer L. Hyperphosphatemia-induced nanocrystals upregulate the expression of bone morphogenetic protein- 2 and osteopontin genes in mouse smooth muscle cells in vitro. Kiney Int. 2011; 79: 414-422.

10) Smith ER, Ford ML, Tomlinson LA, Bodenham E, McMahon L, Farese s, et al. Serum calcification propensity predicts all-cause mortality in predialysis CKD. J Am Soc Nephrol. 2014; 25: 339-348.

11) Dahle DO, Asberg A, Hartmann A, Holdaas H, Bachtler M, Jenssen TG, et al. Serum calcification propensity is a strong and independent determinant of cardiac and allcause mortality in kidney transplant recipients. Am J Transplant. 2016; 16: 204-212.

12) Pasch A, Block GA, Bachtler M, Smith ER, Jahnen-Dechent W, Arampatzis S, et al. Blood calcification propensity, cardiovascular events, and survival in patients receiving hemodialysis in the EVOLVE Trial. Clin J Am Soc Nephrol. 2017; 12: 315322.

13) Bostom A, Pasch A, Madsen T, Roberts MB, Franceschini N, Steubl D, Garimella PS, et al. Serum calcification propensity and fetuin-A: Biomarkers of cardiovascular disease in kidney transplant recipients. Am J Nephrol. 2018: 48: 21-31. 
14) Bundy JD, Cai X, Mehta RC, Scialla JJ, de Boer IH, Hsu Chi-Yuan, et al. Serum Calcification Propensity and Clinical Events in CKD. Clin J Am Soc Nephrol. 2019; 14: $1562-1571$.

15) Chen W, Anokhina V, Dieudonne G, Abramowitz M, Kashyap R, Yan C, et al. Patients with advanced chronic kidney disease and vascular calcification have a large hydrodynamic radius of secondary calciprotein particles. Nephrol Dial Transplant. 2019; 34: 992-1000.

8 16) Lorenz G, Steubl D, Kemmner S, Pasch A, Koch-Sembdner W, Pham D, et al.

9 Worsening calcification propensity precedes all-cause and cardiovascular mortality in $10 \quad$ haemodialyzed patients. Sci Rep. 2017; 7: 13368. 
$1 \quad$ Figure legends

2

3 Fig 1. Relationship between CPP and phosphate (A), corrected calcium (B), eGFR (C).

4 Phosphate and corrected calcium showed significant relationship with CCP, but 5 eGFR did not show.

6

$7 \quad$ Fig 2. Relationship between CPP and Log ACS is shown. There is no significant 8 correlation.

9 


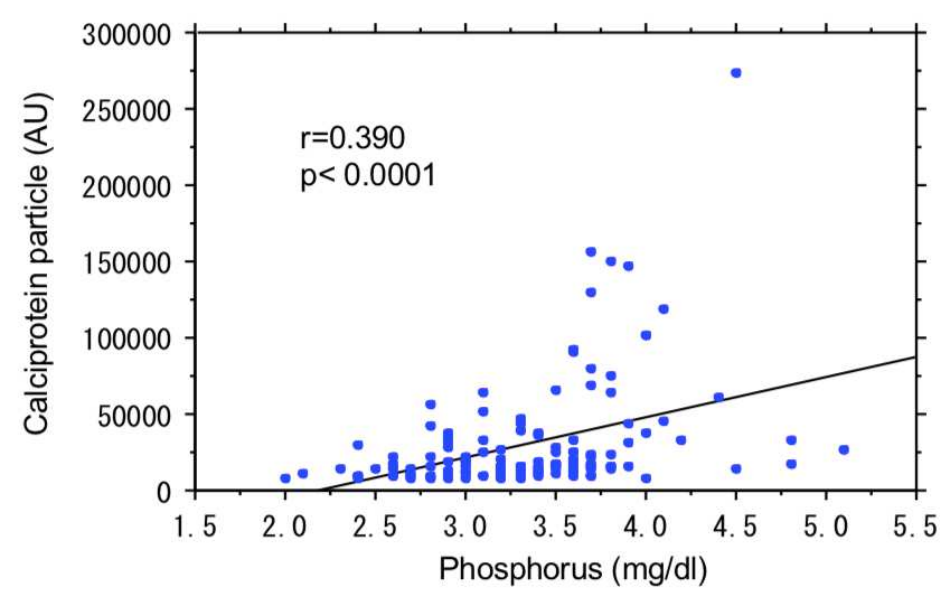

3

4 B

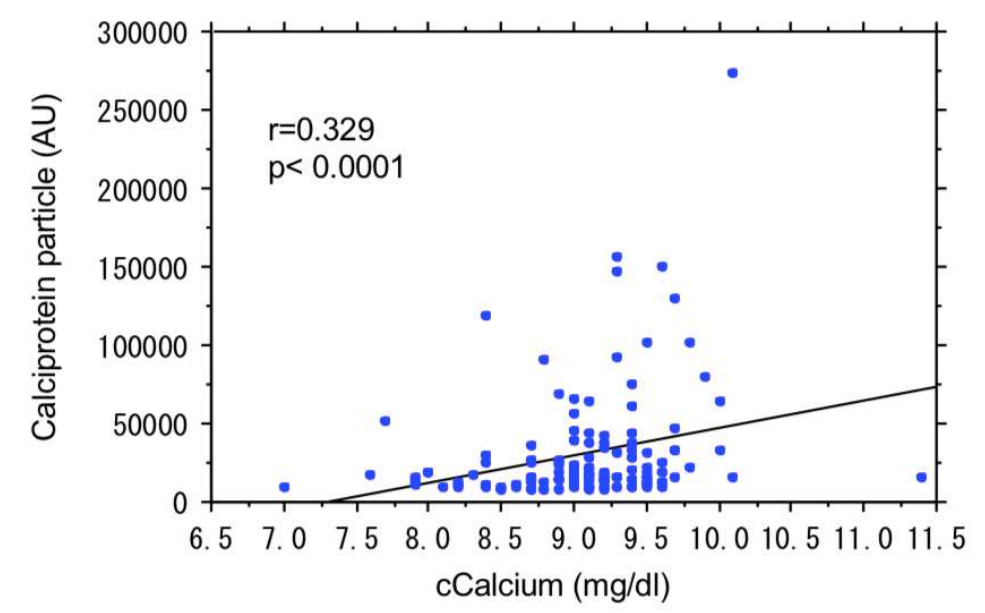

6 C

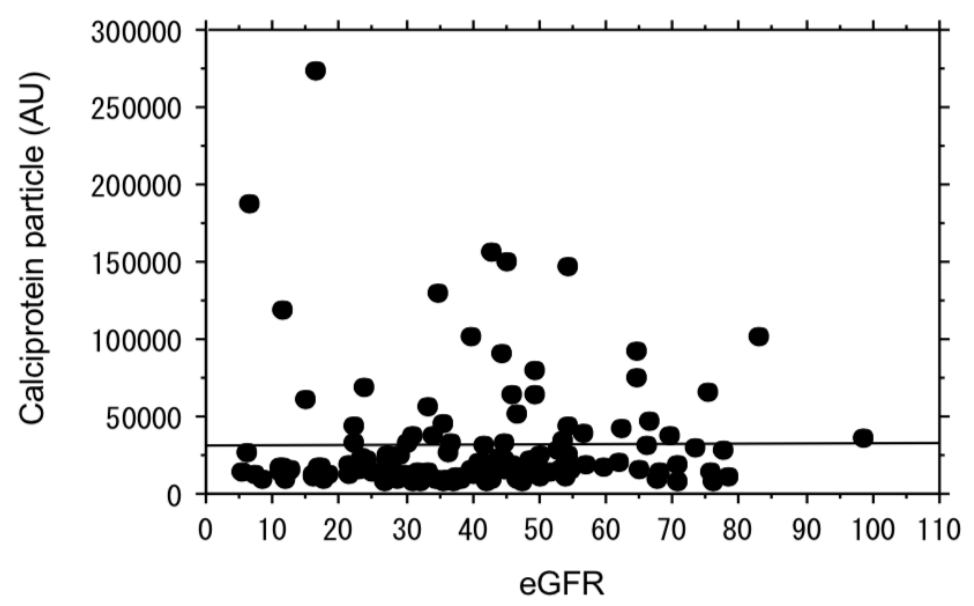

7

8 Figure 1 


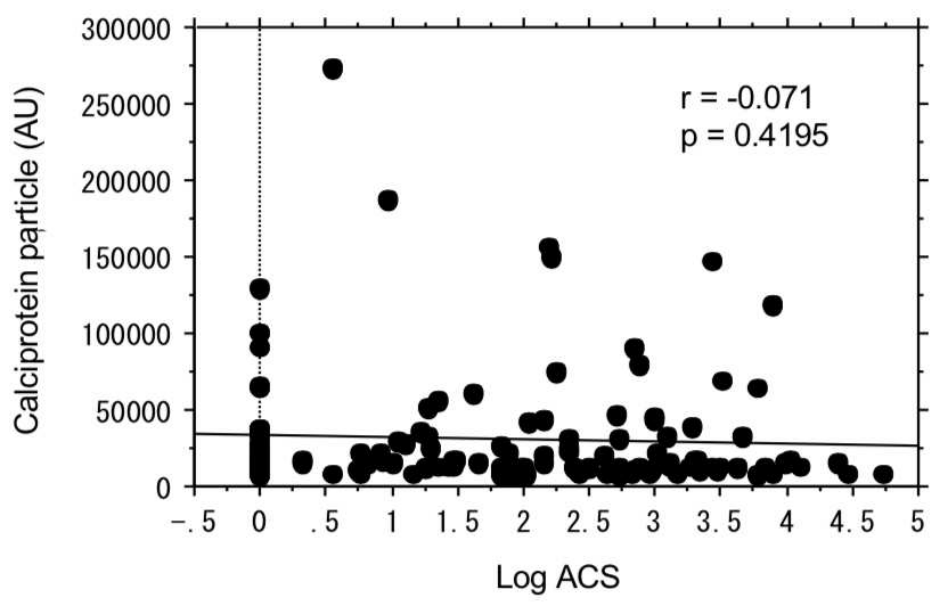

2

$3 \quad$ Figure 2

4 
1 Table 1. Basic characteristics of patients $(n=58)$

Age $70.0 \pm 12.3$

Sex $45(77.5 \%)$

Current smoking

$9(15.5 \%)$

BMI $23.8 \pm 4.0$

History of diabetes $24(41.3 \%)$

History of CVD $17(29.3 \%)$

History of hyperlipidemia $35(60.3 \%)$

History of hypertension $45(77.5 \%)$

Systolic BP (mmHg) $131.7 \pm 17.8$

Diastolic BP (mmHg) $77.7 \pm 9.7$

Total protein $(\mathrm{g} / \mathrm{dl})$ $7.1 \pm 0.6$

Albumin (g/dl)

$4.1 \pm 0.4$

BUN (mg/dl)

$25.2 \pm 11.1$

Creatinine (mg/dl) $1.42(1.10-1.86)$

eGFR (mL/min $\left./ 1.73 \mathrm{~m}^{2}\right)$ 39.7 (24.7-50.1)

corrected calcium $(\mathrm{mg} / \mathrm{dl})$ $9.3 \pm 0.4$ phosphate $(\mathrm{mg} / \mathrm{dl})$ $3.4 \pm 0.6$

$\mathrm{HCO}^{3}-(\mathrm{mmol} / \mathrm{L})$ $24.5 \pm 2.5$

Hemoglobin (g/dl) $13.3 \pm 1.7$

CRP (mg/dl) $0.06(0.03-0.18)$

i-PTH $(\mathrm{pg} / \mathrm{ml})$ 67 (50-105)

1.25( $\left(\mathrm{OH}_{2}\right) \mathrm{VitD} 3$ $50.1 \pm 22.4$

FGF-23 (pg/ml) 63.7 (41.7-115.5)

CPP (AU) 16616 (11894-29152)

Urinary protein (g/gcr)

$0.36(0.09-1.19)$

FE P (\%)

$23.5 \pm 14.7$

ACS $655(0-706)$

3 BMI: body mass index, CVD: cardiovascular disease, BP: blood pressure, CRP: C-reactive 4 protein, i-PTH: intact parathyroid hormone, VitD3: vitamin D3, FEP: fractional excretion 5 of phosphate, ACS: arterial calcification score 
1 Table 2. Univariate and multivariate analysis of relationship between ACS in lower

2 extremities and CKD-MBD markers

\begin{tabular}{|c|c|c|c|c|}
\hline & \multicolumn{2}{|c|}{ univariate } & \multicolumn{2}{|c|}{ multivariate } \\
\hline & $r$ & $p$ & $r$ & $p$ \\
\hline Age & 0.503 & $<0.0001$ & & \\
\hline Smoking & 0.291 & 0.0007 & 0.552 & 0.0004 \\
\hline Diabetes mellitus & 0.388 & $<0.0001$ & 0.790 & 0.0009 \\
\hline Systolic BP & 0.312 & 0.0003 & 0.017 & 0.0064 \\
\hline Albumin & -0.060 & 0.4943 & & \\
\hline Creatinine & 0.196 & 0.0238 & & \\
\hline eGFR & -0.295 & 0.0005 & & \\
\hline $\mathrm{HCO}^{3}-$ & -0.162 & 0.0634 & & \\
\hline cCalcium & 0.019 & 0.8307 & & \\
\hline Phosphate & 0.021 & 0.8074 & & \\
\hline Log CRP & 0.072 & 0.4102 & & \\
\hline i-PTH & 0.034 & 0.6983 & & \\
\hline Hemoglobin & -0.178 & 0.0406 & -0.018 & 0.7315 \\
\hline Urinary protein & 0.172 & 0.0497 & 0.096 & 0.0766 \\
\hline $1.25\left(\mathrm{OH}_{2}\right) \mathrm{VitD} 3$ & -0.141 & 0.1072 & & \\
\hline FE P & 0.175 & 0.0467 & 0.004 & 0.9971 \\
\hline FGF-23 & 0.096 & 0.2825 & & \\
\hline Log CPP & -0.071 & 0.4195 & & \\
\hline
\end{tabular}

4 ACS: arterial calcification score, BP: blood pressure, CRP: C-reactive protein, i-PTH:

5 intact parathyroid hormone, VitD3: vitamin D3, FE P: fractional excretion of phosphate 6 
1 Table 3. Relationship between basic factors and change of ACS for one year

\begin{tabular}{|c|c|c|}
\hline & $r$ & $p$ \\
\hline Age & 0.089 & 0.5102 \\
\hline Smoking & -0.134 & 0.3161 \\
\hline $\mathrm{DM}$ & -0.041 & 0.7608 \\
\hline Systolic BP & 0.041 & 0.7607 \\
\hline Albumin & 0.150 & 0.2620 \\
\hline Creatinine & -0.011 & 0.9334 \\
\hline eGFR & -0.007 & 0.9557 \\
\hline $\mathrm{HCO}^{3}-$ & -0.042 & 0.7549 \\
\hline cCalcium & -0.030 & 0.8257 \\
\hline Phosphate & 0.195 & 0.1434 \\
\hline Log CRP & 0.067 & 0.6162 \\
\hline i-PTH & -0.038 & 0.7805 \\
\hline Hemoglobin & -0.044 & 0.7450 \\
\hline Urinary protein & -0.159 & 0.2342 \\
\hline $1.25\left(\mathrm{OH}_{2}\right)$ VitD3 & 0.043 & 0.7505 \\
\hline FE P & -0.090 & 0.5031 \\
\hline FGF-23 & 0.027 & 0.8440 \\
\hline Log CPP & 0.073 & 0.5860 \\
\hline
\end{tabular}

3 ACS: arterial calcification score, BP: blood pressure, CRP: C-reactive protein, i-PTH:

4 intact parathyroid hormone, VitD3: vitamin D3, FE P: fractional excretion P, 5 6 
1 Table 4. Univariate and multivariate analysis of relationship between rate of change of

2 ACS and other factors

3

\begin{tabular}{|l|c|c|c|c|}
\hline & \multicolumn{2}{|c|}{ univariate } & \multicolumn{2}{|c|}{ multivariate } \\
\hline Change rate of Creatinine & $r$ & $p$ & $\mathrm{r}$ & $\mathrm{P}$ \\
\hline Change rate of eGFR & -0.005 & 0.9712 & & \\
\hline Change rate of FGF-23 & -0.042 & 0,7544 & \\
\hline Change rate of CPP & 0.046 & 0.7411 & & \\
\hline Change rate of i-PTH & 0.292 & 0.0258 & 0.298 & 0.0144 \\
\hline Change rate of $1.25\left(\mathrm{OH}_{2}\right)$ VitD3 & -0.124 & 0.3592 & & \\
\hline
\end{tabular}

4 ACS: arterial calcification score, i-PHT: intact parathyroid hormone, VitD3: vitamin D3 5 
Figures
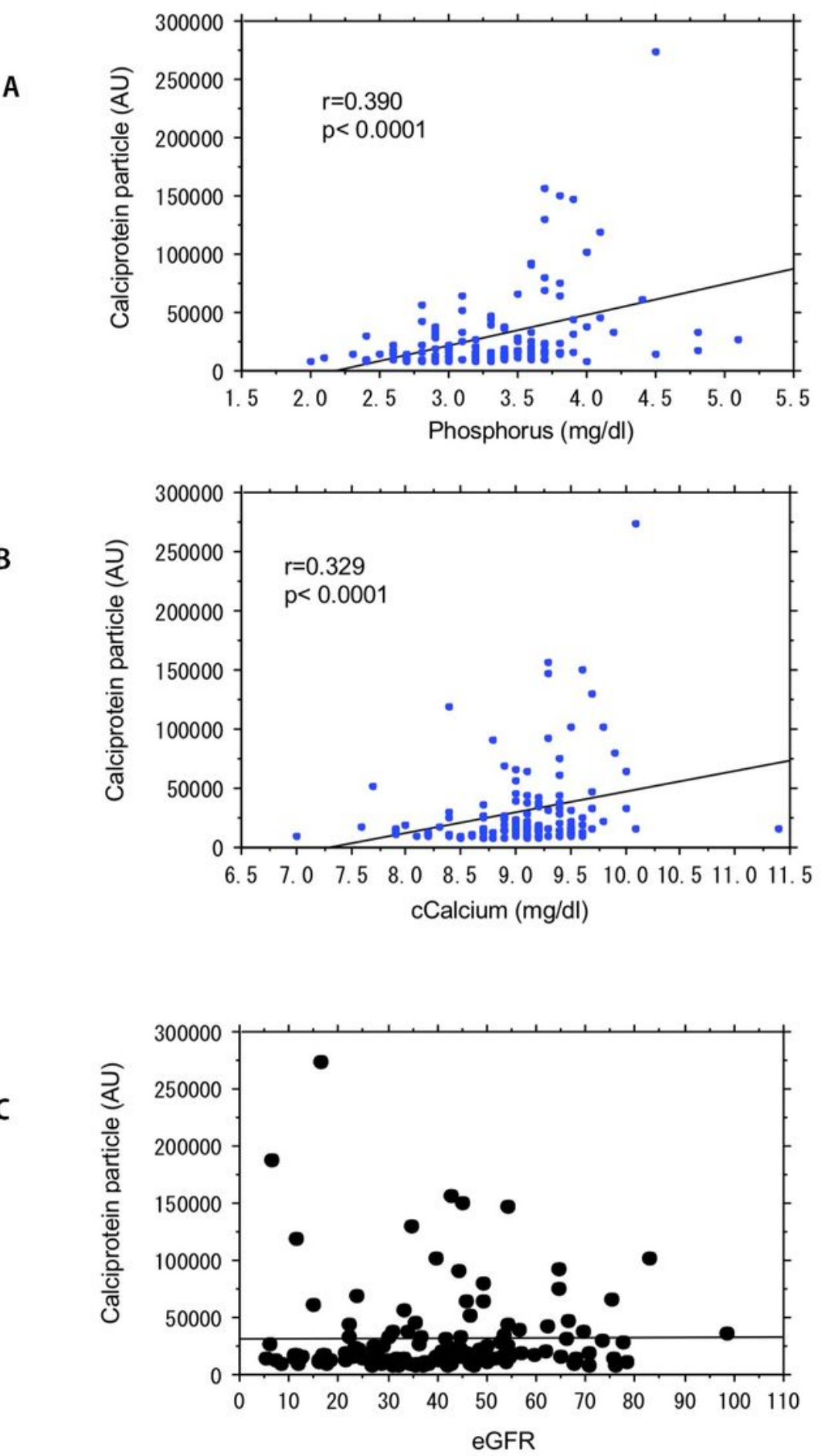

Figure 1

Relationship between CPP and phosphate (A), corrected calcium (B), eGFR (C).Phosphate and corrected calcium showed significant relationship with CCP, but eGFR did not show. 


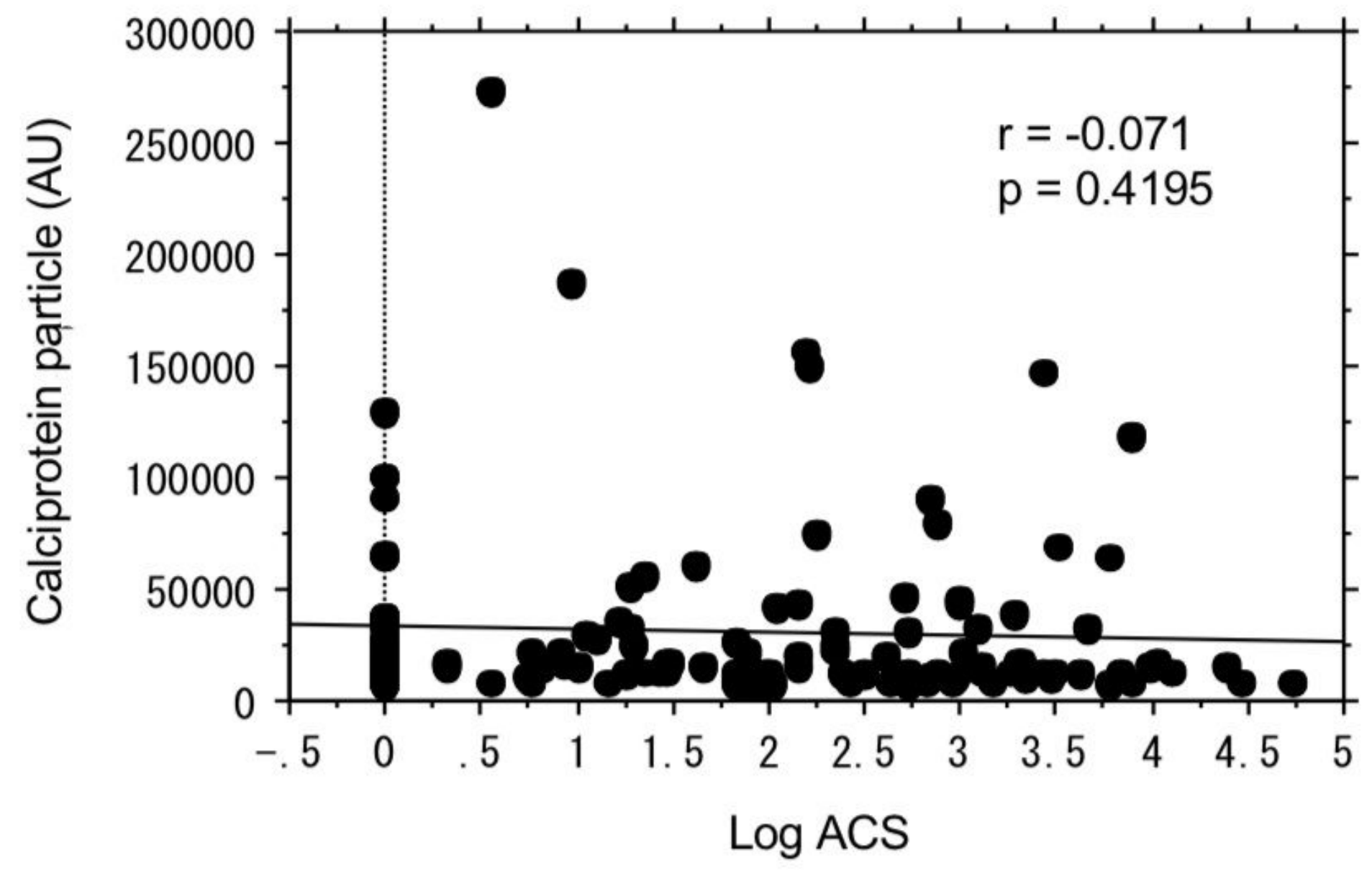

Figure 2

Relationship between CPP and Log ACS is shown. There is no significant correlation. 